\title{
Evaluación de la influencia del estado nutricional en el control de diabetes mellitus tipo 2
}

\section{Evaluation about the influence of the nutritional state in the control of Diabetes mellitus type 2}

\author{
Isabel Rojas-Padilla ${ }^{1}$ (D), Diana Zambrano-Ríos ${ }^{2}$ (D), Andrés Matta-Miramar ${ }^{3}$ (D) \\ 1. Escuela Nacional del Deporte. Cali, Colombia. Correo: isabel.rojas@endeporte.edu.co - http://orcid.org/0000-0001-8994-6529 \\ 2. Escuela Nacional del Deporte. Cali, Colombia. Correo: diana.zambrano@endeporte.edu.co - http://orcid.org/0000-0002-8636-1629 \\ 3. Escuela Nacional del Deporte. Cali, Colombia. Correo: andres.matta@endeporte.edu.co - http://orcid.org/0000-0002-9637-1812 \\ Tipología: Artículo de investigación científica y tecnológica \\ Para citar este artículo: Rojas-Padilla IC, Zambrano-Ríos DC, Matta-Miramar AJ. Evaluación de la influencia del estado nutricional en el control de la diabetes mellitus tipo 2. \\ Duazary. 2020 abril- junio; 17(2): 10 - 19. Doi: http://dx.doi.org/10.21676/2389783X.3232

\section{RESUMEN}

Palabras clave: estado estilo de vida; diabetes mellitus. nutricional;

La diabetes es una enfermedad no transmisible cuya importancia epidemiológica radica en el aumento exponencial de la morbilidad y la mortalidad en todo el mundo. Se pretendió determinar la asociación entre la clasificación nutricional de los pacientes con diabetes y la influencia en el control de la patología mediante un estudio descriptivo transversal en 237 participantes con diabetes, hombres y mujeres mayores de edad pertenecientes a un programa de control en dos entidades que brindan servicios de salud. El análisis estadístico de los datos se realizó con el software SPSS versión 25, con tablas de contingencia y prueba de chi cuadrado, calculando el valor de p con un intervalo de confianza del $95 \%$. Se encontró que el 49,36\% de los participantes tenía un nivel de hemoglobina glicosilada superior al 7\%, el 51,89\% con lipoproteínas de baja densidad mayor a $100 \mathrm{mg} / \mathrm{dl}$, el 63,71\% sin practicar actividad física y el $68,3 \%$ en exceso de peso. Los hábitos de vida saludable, como sana alimentación y ejercicio regular, pueden ser determinantes en el control patológico de la diabetes mellitus tipo 2 pues influyen en la pérdida de peso y sus comorbilidades, factores importantes en el estado de salud de este tipo de pacientes.

\section{ABSTRACT}

Keywords: Nutritional Status; life Style; Diabetes Mellitus.
The diabetes is one of the non-communicable diseases and its epidemiological importance lies in the exponential increase in morbidity and mortality in all over the world. The aim was to determine the association between the nutritional classification of patients with diabetes and the influence in the control of the pathology. Descriptive and cross-sectional study done in 237 elderly men and women with diabetes, belonging to a control program in two entities that provide health services. Statistical analysis of the data was done using SPSS software version 25, performing contingency tables and chi-squared test, calculating the $P$ value with a confidence interval of $95 \%$. It was found $49.36 \%$ of the participants with the glycosylated hemoglobin level higher than 7\%, 51.89\% with low density lipoprotein out of goal, $63.71 \%$ who did not practice any physical activity and $68.3 \%$ in excess weight. As a conclusion, healthy life styles such as healthy food and regular practice of physical exercise can be decisive in the pathological control of diabetes mellitus type 2 since they influence in the loss of weight and comorbidities, relevant factors in this kind of patients' nutritional status. 


\section{INTRODUCCIÓN}

Las enfermedades no transmisibles (ENT) son un problema de salud pública a nivel mundial, responsables de un alto índice de muerte y que demandan la detección de factores de riesgo con el fin de trabajar en la promoción y prevención, disminuyendo así la prevalencia de este tipo de patologías. De acuerdo con la Organización Mundial de la Salud (OMS), de los 57 millones de muertes reportadas en el 2008, 36 millones (63\%) se debieron a ENT, entre ellas enfermedades cardiovasculares, diabetes, cáncer y enfermedades respiratorias crónicas ${ }^{1}$. "Las ENT se deben en gran medida a cuatro factores de riesgo comportamentales que se han afianzado de forma generalizada como parte de la transición económica, los rápidos procesos de urbanización y los modos de vida del siglo XXI: el consumo de tabaco, las dietas malsanas, la inactividad física y el uso nocivo del alcohol" 1 .

Para el 2015 había 415 millones de personas en el mundo que padecían diabetes; sin embargo, para el 2040 se estima que esta cantidad aumentará un $35 \%$, a 642 millones $^{2}$. La diabetes mellitus (DM) es un problema de salud que afecta el mundo entero y que no discrimina nivel económico, raza, género, cultura ni edad. Anteriormente, la DM tipo 2 solía ser una patología de adultos y poco frecuente; no obstante, en la actualidad es la más común, y su incidencia está aumentando en la población más joven ${ }^{2}$.

En el 2017 la OMS ${ }^{1}$ estimó que, en el mundo, la glucosa alta en sangre es el tercer factor principal de riesgo para la mortalidad prematura, después de la presión arterial alta y el consumo de tabaco. Por su parte, la Federación Internacional del Diabetes (FDI) estima que 1 de cada 11 adultos tiene diabetes, y de igual manera señala que 1 de cada 2 adultos con diabetes está sin diagnosticar. Esto representa el $12 \%$ del gasto en salud mundial que se destina a la diabetes $^{2}$, panorama bastante devastador para la salud pública. En Colombia, la OMS (2014) ${ }^{3}$ estimó la prevalencia de DM en $8,5 \%$.

Entre los factores de riesgo asociados al desarrollo de esta patología se encuentran: exceso de peso, inactividad física, dieta inadecuada, antecedentes familiares, edad avanzada y otras enfermedades. Aunque algunos factores no se pueden modificar, como son la edad y la historia familiar del individuo, existen otros, como la alimentación y la actividad física, que pueden ser trabajados en pro de evitar este padecimiento e incluso como factor protector ${ }^{4}$.

En la actualidad las personas diagnosticadas con diabetes aumentan en un porcentaje considerable, $y$ aunque esta patología no tiene cura, es una enfermedad tratable en cuyo control la intervención médica y nutricional desempeña un papel muy importante. Esto último es lo que, en otras palabras, se denomina estado nutricional, refiriéndose a él como el estado de salud con relación a la alimentación reflejado en el índice de masa corporal (IMC), el cual se clasifica numéricamente en rangos: menor a 18,5 (bajo peso), entre 18,6 y 24,9 (normopeso), entre 25 y 29,9 (sobrepeso) y mayor de 30 (obesidad) (Resolución 2464 del 14 de junio de 2016).

Adicionalmente, el hecho de que una persona que padece diabetes se encuentre con sobrepeso y obesidad genera una complicación y factor de riesgo para un descontrol en la patología, dificultando de cierta manera que otros indicadores como la hemoglobina glicosilada (HbA1c), la tensión arterial $(T / A)$, el perímetro abdominal (PA) y el colesterol de baja densidad en sangre (LDL) se ubiquen dentro de las metas establecidas por diferentes entes de control. Entre estos, la Asociación Americana de Diabetes ${ }^{5}$ estima como meta de $\mathrm{HbA} 1 \mathrm{c}$ un máximo de $7 \%$, una $\mathrm{T} / \mathrm{A}$ que no sobrepase $140 / 90 \mathrm{mg} / \mathrm{dl}$ y un LDLc menor de $100 \mathrm{mg} / \mathrm{dl}$; asimismo, el Ministerio Colombiano de Salud y Protección Social, en su Resolución 2465 del 14 de junio de 2016, determinó evaluar la presencia de riesgo cardiovascular mediante el perímetro abdominal mayor a $94 \mathrm{~cm}$ para hombres y a $88 \mathrm{~cm}$ para mujeres. Por otra parte, para la población mayor de 18 años, la $\mathrm{OMS}^{4}$ recomienda práctica de actividad física de un mínimo de 150 minutos a la semana distribuidos en actividad moderada o vigorosa (considerando individualidades), teniendo en cuenta este tiempo como meta de la variable, siendo este otro ítem por considerar dentro de aquellas estrategias que ayudarían a controlar la DM2.

De esta manera, el objetivo del presente estudio fue 
determinar la asociación entre el estado nutricional de los pacientes y el control de la patología teniendo en cuenta las metas estimadas en el párrafo anterior para cada variable y la relación de estas con su clasificación nutricional de acuerdo al IMC, ya que son mediadas por el estilo de vida -alimentación y ejercicio- y su diagnóstico e intervención puede prevenir las complicaciones propias de la DM2.

\section{MATERIALES Y MÉTODOS}

\section{Tipo de investigación}

Estudio descriptivo de tipo transversal. Los datos fueron obtenidos en la consulta nutricional de un grupo de pacientes.

\section{Población y muestra}

La población original estuvo compuesta por 315 pacientes con diabetes, hombres y mujeres mayores de 18 años pertenecientes al régimen contributivo que hacían parte del programa de control en dos entidades prestadoras de servicios de salud en Cali, Colombia. Como criterios de exclusión se consideraron pacientes que asistieron por primera vez a cita de nutrición, gestantes, menores de edad y pacientes con diabetes tipo I. Finalmente, la muestra estuvo constituida por 237 personas.

\section{Instrumentos y procedimiento}

La información se obtuvo durante tres meses de consulta en jornadas de cuatro horas tres veces por semana. Los datos se recolectaron mediante una formulario de 12 ítems diligenciados por la nutricionista en los que se incluyeron número de identidad, edad, género, diagnóstico médico adicional al de diabetes, última cita de control previo a la del momento, valores de hemoglobina glicosilada (A1c), tensión arterial (TA), colesterol LDL (LDLC), realización de actividad física, medidas antropométricas de peso, talla y perímetro abdominal, además de preguntas de rutina durante la consulta como frecuencia de consumo de ciertos alimentos y horarios de comidas. Esta información se obtuvo bajo el protocolo ya establecido por la nutricionista, de manera que su recolección no implicó tiempo extra o por fuera de la consulta.

Las variables bioquímicas tales como LDLc fueron tomadas a partir de suero extraído de sangre periférica del paciente en ayuno. La cuantificación de los niveles de LDLc se realizó por método directo utilizando espectrofotometría de absorción a una temperatura de 37 ㅇ y una longitud de onda de 546 $\mathrm{nm}$, siguiendo el inserto de los reactivos por la marca comercial BioSystems. Para esta prueba se emplearon controles y calibradores, externos e internos, normales y anormales, los cuales estuvieron dentro de los rangos aceptados para cada uno de ellos, y se utilizó el equipo automatizado BS120 de la marca comercial Mindray.

Para la cuantificación de los niveles de A1c se utilizó plasma extraído de sangre venosa periférica colectada en tubo tapa lila con anticoagulante EDTA, de la cual se tomó una alícuota con el fin de preparar el hemolizado para realizar la cuantificación mediante el método turbidimétrico con látex, siguiendo el protocolo del fabricante del reactivo BioSystems. Para esta prueba se emplearon controles, externos e internos, normales y anormales, los cuales estuvieron dentro de los rangos aceptados para cada uno de ellos, y se utilizó el equipo automatizado A25 de la marca comercial BioSystems.

Para la medición de la tensión arterial fue necesario indicar al paciente que adoptara un estado de reposo por 5 minutos previo a la toma. Finalmente, se procedió a tomar la tensión arterial con el paciente sentado con la espalda recta en un asiento con buen soporte, el brazo izquierdo descubierto apoyado a la altura del corazón, piernas sin cruzar y pies apoyados cómodamente sobre el suelo. Las medidas se tomaron utilizando un esfigmomanómetro manual y el manómetro de mercurio, los cuales se calibraron no menos de tres meses antes de la toma de la muestra.

Finalmente, para la toma de medidas antropométricas de peso, talla y perímetro abdominal se utilizó una báscula de columna inalámbrica con estadiómetro integrado Seca 703s, y el perímetro abdominal se tomó con una cinta métrica Lufkin 606.

\section{Análisis estadístico}

Se realizó el análisis estadístico de los datos utilizando el software SPSS versión 25 elaborando tablas de contingencia y prueba de chi cuadrado. El 
valor $\mathrm{p}$ se calculó con un intervalo de confianza del $95 \%$.

\section{Declaración sobre aspectos éticos}

Todos los participantes fueron debidamente informados y firmaron consentimiento informado para que sus datos hicieran parte del estudio. La investigación fue aprobada por el comité ético de investigaciones de la Escuela Nacional del Deporte (Cali) mediante acta de aprobación END 4,1-18-04 con fecha de febrero del 2018. Al ser una investigación con seres humanos, se adaptó a las normas establecidas en la Declaración de Heinsinki de 1975 y la Resolución 8430 de 1993 de Ministerio de Salud de Colombia.

\section{RESULTADOS}

Se halló población heterogénea en género, representada en su mayoría por adultos medios y mayores, correspondientes a edades por encima de los 40 años y clasificados nutricionalmente en su mayoría con exceso de peso. Para esta clasificación se tuvo en cuenta el índice de masa corporal contemplado en la Resolución 2465 del 2016 del Ministerio de Salud y Protección Social (tabla 1).

Tabla 1. Clasificación nutricional y demografía.

\begin{tabular}{|c|c|c|c|c|}
\hline Características & $\begin{array}{c}\text { Bajo peso } \\
\mathbf{n}(\mathbf{1 7}) \\
\text { Número (\%) }\end{array}$ & $\begin{array}{c}\text { Normo peso } \\
\mathbf{n}(\mathbf{5 8 )} \\
\text { Número (\%) }\end{array}$ & $\begin{array}{c}\text { Sobrepeso } \\
\mathbf{n}(\mathbf{7 2}) \\
\text { Número (\%) }\end{array}$ & $\begin{array}{c}\text { Obesidad } \\
\mathbf{n}(\mathbf{9 0}) \\
\text { Número (\%) }\end{array}$ \\
\hline Género & & & \\
\hline Masculino & $8(47,1)$ & $27(46,6)$ & $46(63,9)$ & $39(43,3)$ \\
\hline Femenino & $9(52,9)$ & $31(53,4)$ & $26(36,1)$ & $51(56,7)$ \\
\hline \multicolumn{5}{|c|}{ Edad } \\
\hline Adulto joven (20-39) & $5(29,4)$ & $9(15,5)$ & $13(18,1)$ & $3(3,3)$ \\
\hline Adulto medio (40-59) & $7(41,2)$ & $24(41,4)$ & $38(52,8)$ & $32(35,6)$ \\
\hline Adulto mayor (>60) & $5(29,4)$ & $25(43,1)$ & $21(29,2)$ & $55(61,1)$ \\
\hline
\end{tabular}

* La clasificación de edades (adulto joven, medio y mayor) corresponde a la determinada por la OMS.

En este estudio se encontró que 117 de 237 participantes presentaron un nivel de hemoglobina glicosilada superior a $7 \%$. En el $52 \%$ de los casos se evidenció un aumento de la lipoproteína de baja densidad, el $64 \%$ no realizaban ejercicio, y el $68,3 \%$ de los participantes estaban en condición de exceso de peso, ya fuera sobrepeso u obesidad (tabla 2 y tabla 3).

Este acercamiento investigativo a los pacientes muestra que el $35 \%$ de ellos no realizan actividad física, incumpliendo la meta pactada por la OMS para dicha población. Adicional a ello, todas las personas sedentarias son obesas con un valor de $p$ de 0,049 . También se evidencia, por un lado, el riesgo cardiovascular relacionado en un alto porcentaje en personas con exceso de peso debido a la toma de perímetro abdominal para obtener el valor y, por otro lado, la tensión arterial con metas no cumplidas en un porcentaje considerable. 
Tabla 2. Estadísticos descriptivos de las variables.

\begin{tabular}{|c|c|c|c|c|c|}
\hline Característica & $\begin{array}{c}\text { Valor } \\
\text { Mínimo }\end{array}$ & $\begin{array}{c}\text { Valor } \\
\text { máximo }\end{array}$ & Media & $\begin{array}{c}\text { Desviación } \\
\text { estándar }\end{array}$ & Kurtosis \\
\hline $\begin{array}{c}\text { Hemoglobina glicosilada } \\
\text { A1c\% }\end{array}$ & 5,4 & 12,1 & 7,5 & 1,26 & 0,48 \\
\hline Tensión arterial sistólica & 100 & 170 & 138,4 & 16,82 & $-0,70$ \\
\hline Tensión arterial diastólica & 60 & 100 & 86,8 & 9,13 & 1,37 \\
\hline Colesterol LDL & 57 & 198 & 109,1 & 34,03 & $-0,35$ \\
\hline Perímetro abdominal & 71,5 & 136 & 98 & 12,24 & $-0,44$ \\
\hline
\end{tabular}

Tabla 3. Relación de clasificación nutricional con factores de riesgo en control de la diabetes.

\begin{tabular}{|c|c|c|c|c|c|}
\hline \multirow[b]{2}{*}{ Características } & \multicolumn{5}{|c|}{ Clasificación nutricional } \\
\hline & $\begin{array}{c}\text { Bajo peso } \\
\text { n (17) } \\
\text { Número (\%) }\end{array}$ & $\begin{array}{c}\text { Normo peso } \\
\text { n (58) } \\
\text { Número (\%) }\end{array}$ & $\begin{array}{c}\text { Sobrepeso } \\
\text { n (72) } \\
\text { Número (\%) }\end{array}$ & $\begin{array}{c}\text { Obesidad } \\
\text { n (90) } \\
\text { Número (\%) }\end{array}$ & Valor de $p$ \\
\hline \multicolumn{5}{|c|}{ Actividad física } & 0,049 \\
\hline No cumple meta & $3(17,6)$ & $12(20,7)$ & $52(72,2)$ & $84(93,3)$ & \\
\hline Cumple meta & $14(82,4)$ & $46(79,3)$ & $20(27,8)$ & $6(6,7)$ & \\
\hline \multicolumn{5}{|c|}{ Colesterol LDL } & 0,048 \\
\hline No cumple meta & $7(41,2)$ & $3(5,2)$ & $38(52,8)$ & $75(83,3)$ & \\
\hline Cumple meta & $10(48,8)$ & $55(94,8)$ & $34(47,2)$ & $15(16,7)$ & \\
\hline \multicolumn{5}{|c|}{ Hemoglobina glicosilada } & 0,044 \\
\hline No cumple meta & $3(17,6)$ & $6(10,3)$ & $32(44,4)$ & $76(84,4)$ & \\
\hline Cumple meta & $14(82,4)$ & $52(89,7)$ & $40(55,6)$ & $14(15,6)$ & \\
\hline \multicolumn{5}{|c|}{ Riesgo cardiovascular (perímetro abdominal) } & 0,049 \\
\hline Sin riesgo & $13(76,5)$ & $47(81,0)$ & $8(11,1)$ & 0() & \\
\hline Con riesgo & $4(23,5)$ & $11(19,0)$ & $64(88,9)$ & $90(100.0)$ & \\
\hline \multicolumn{5}{|c|}{ Tensión arterial } & 0,054 \\
\hline No cumple meta & $11(35,3)$ & $14(24.1)$ & $55(76,4)$ & $75(83,3)$ & \\
\hline Cumple meta & $6(64,7)$ & $44(75,9)$ & $17(23,6)$ & $15(16,7)$ & \\
\hline
\end{tabular}

\section{DISCUSIÓN}

Si bien es cierto que la frecuencia de casos de diabetes enciende alarmas a nivel de salud pública, el gran aspecto por considerar dentro de la relevancia epidemiológica de esta patología es la morbilidad y mortalidad que suma a las estadísticas mundiales ${ }^{6}$. Las características más encontradas en estos pacientes son la malnutrición por exceso de peso ${ }^{7}$, dato que no solo incrementa en pacientes con diabetes, sino en la población en general, como lo revelan las encuestas de vigilancia nutricional en algunos países de América latina: Perú $(52 \%)^{8}$, Colombia $(56 \%)^{9}$, Chile $(64 \%)^{10}$ y México $(72,5 \%)^{11}$ para nombrar algunos.

Dentro de los avances más significativos en el estudio de riesgos de salud asociados al exceso de peso se encuentran investigaciones enfocadas en la 
grasa corporal; más específicamente, en la predominancia de esta a nivel abdominal ${ }^{12}$. A finales de los cuarenta Vague sugirió que la predominancia de grasa abdominal podría contribuir al aumento de riesgo de enfermedades crónicas como la diabetes ${ }^{13}$, hipótesis que se ha confirmado a lo largo de la historia. Además, diferentes investigaciones han ayudado a esclarecer la tasa de morbilidad y mortalidad por aumento de peso y otros factores desencadenantes tales como el aumento de la lipoproteína de baja densidad LDL, diabetes, hipertensión arterial y sedentarismo, que se incrementan día a día a nivel mundial ${ }^{14}$. Todos estos factores inciden en el estado nutricional de una persona, y aún más si su diagnóstico es de diabetes, en quienes el sobrepeso y sobre todo la obesidad generan alarma en el control y tratamiento de dicha enfermedad, pues son desencadenantes de otros factores que complican el control y generan desmejora en la calidad de vida.

Se estima que 97 millones de estadounidenses adultos padecen exceso de peso, aumentando generosamente el riesgo de morbilidad por enfermedades crónicas como accidentes cerebrovasculares, hipertensión, cáncer, enfermedad coronaria y, por supuesto, diabetes mellitus $^{15}$, factor que puede terminar en fallecimientos, pues el peso corporal aumentado está asociado a mortalidad. Adicionalmente, los individuos obesos pueden enfrentarse a discriminación y estigmatización desde su comunidad, lo que incrementa factores de riesgo para la salud como depresión. De esta manera, el sobrepeso y la obesidad como factor de riesgo en la prevención de la mortalidad constituyen hoy en día un importante reto de salud pública, no solo en los países industrializados, sino también en los países en desarrollo.

Los hallazgos de este estudio muestran A1c, TA y LDLc altos y PA aumentado, los cuales cruzan con diagnóstico de obesidad, nada diferente a las proyecciones de la OMS y los resultados de estudios previos $^{16-19}$

Respecto al sedentarismo, en el año 2002 la Organización Mundial de la Salud (OMS) informó que los estilos de vida caracterizados por este eran una de las diez causas principales de mortalidad y discapacidad en el mundo ${ }^{20}$. Ocho años después, se encontró que mil millones de personas padecen malnutrición por exceso, es decir, están en sobrepeso u obesidad a causa de enfermedades crónicas debido a dietas insanas y falta de actividad física ${ }^{21}$. De acuerdo con la OMS 22 , "En la actualidad, se estima que el sedentarismo es la causa principal de aproximadamente el $25 \%$ del cáncer de colon y mama; $27 \%$ de los casos de diabetes mellitus tipo 2 y de $30 \%$ de los pacientes con enfermedad coronaria, representando la cuarta causa de mortalidad mundial".

En nuestro país, la Encuesta Nacional de Salud de $2007^{23}$ y la Encuesta Nacional de la Situación Nutricional en Colombia (2010) $)^{9}$ mostraron prevalencias de sedentarismo que varían del $70 \%$ al $85 \%$ en los hombres y las mujeres respectivamente. Otras observaciones muestran que el sedentarismo está ligado al proceso aterosclerótico ${ }^{24}$, a la predisposición de obesidad ${ }^{25}$ y a la dislipidemia ${ }^{26}$, hallazgos muy relacionados con el presente estudio, en el cual 136 de 162 pacientes con diabetes y exceso de peso son sedentarios.

Una consecuencia más de la diabetes es el síndrome metabólico, causa de complicaciones cardiovasculares, renales y cerebrovasculares en estos pacientes ${ }^{27}$. Así se conjugan variables tenidas en cuenta en este estudio, entre las cuales la creciente prevalencia de hipertensión debida a dietas malsanas y sedentarismo aporta preocupación al diagnóstico ${ }^{28}$. Estos resultados coinciden además con otras publicaciones ${ }^{29-30}$ en las cuales se señala riesgo de complicación de diabetes por presión arterial alta, sin dejar de lado la influencia del perímetro abdominal aumentado en esta patología para consecuentes comorbilidades.

Los principales factores de riesgo para desarrollar enfermedad cardiovascular, según el estudio de Framingham et al., son: la edad (mayor de 35 años), el sexo (masculino), la presión arterial sistólica alta (igual o mayor a $120 \mathrm{mmHg}$ con medicación, igual o mayor a $130 \mathrm{mmHg}$ sin medicación), el tabaquismo (fumar) y la dislipidemia ${ }^{3,31}$. Así mismo, los factores de riesgo cardiovascular se clasifican como modificables y no modificables de acuerdo a las posibilidades de intervención: son no modificables la edad, la herencia y el sexo; y los modificables son aquellos que se pueden intervenir de alguna manera a través de medidas de prevención primaria o 
secundaria tales como la presión arterial elevada, el tabaquismo, la dislipidemia y la diabetes ${ }^{32}$. El presente estudio evidencia que los adultos con edades comprendidas entre 40-59 y los adultos mayores de 60 años son los que presentan mayores tasas de obesidad, aumento del colesterol LDL, hemoglobina glicosilada $>7 \%$, prevalencia de tensión arterial alta, perímetro abdominal aumentado y una tasa menor de realización de actividad física, lo que muestra que existe un riesgo alto de sumar a su diabetes y obesidad una enfermedad cardiovascular, afectando aún más su calidad de vida.

Este estudio sirve como punto de partida para evidenciar la necesidad de realizarse una intervención en investigaciones futuras para modificar el comportamiento y las variables modificables, disminuyendo con ello el riesgo de enfermedad cardiovascular, enfermedades crónicas no transmisibles e inclusive la muerte. Esto representa un punto de suma importancia para los organismos de salud locales, departamentales y nacionales puesto que se podrían implementar políticas de salud pública que contribuyan a la disminución de la morbi-mortalidad de nuestra población asociada a enfermedades crónicas no transmisibles.

\section{CONCLUSIÓN}

Los hábitos de vida saludable como alimentación sana, balanceada, adecuada y variada, además de la práctica regular de ejercicio físico, son determinantes en el estado de salud de los pacientes con diabetes mellitus tipo 2, ya que pueden generar un adecuado control en peso, hemoglobina glicosilada, colesterol, tensión arterial y glucosa en sí.

De acuerdo a las características encontradas en la población participante y a los factores asociados con el riesgo cardiovascular, es posible realizar intervenciones futuras para cambiar el comportamiento y las variables modificables, disminuyendo con ello el riesgo de enfermedad cardiovascular, enfermedades crónicas no transmisibles e inclusive la muerte. Esto representa un punto de suma importancia para los organismos de salud locales, departamentales y nacionales puesto que se podrían implementar políticas de salud pública que contribuyan a la disminución de la morbi-mortalidad de nuestra población asociada a enfermedades crónicas no transmisibles.

Adicionalmente, se debe enfocar la intervención en particular en los asistentes de sexo femenino. De acuerdo a los resultados de este estudio, se puede concluir que, como se ha establecido en otros estudios, no amerita hacerse un seguimiento para riesgo cardiovascular antes de los 39 años debido a que el riesgo es muy bajo y no hay sustento médico científico que justifique este recurso.

Desde los 40 años para nuestra población, además de realizarse exámenes de tamizaje y seguimiento del riesgo cardiovascular, es recomendable establecer el riesgo cardiovascular a fin de profundizar estudios en quien tiene riesgo cardiovascular medio-alto. De esta forma debería ser posible realizar tratamiento secundario a fin de evitar eventos que lleven a desenlaces no deseados que representen riesgo mayor para la salud de la población y desgaste económico para las instituciones de salud tanto públicas como privadas en el país.

En el momento de crear una estrategia que genere impacto en este tipo de pacientes, es importante encontrar en ellos mismos y de forma muy natural las necesidades que presentan, las dificultades a las que se enfrentan, sus expectativas y hasta sus dudas respecto a su tratamiento, y una cita de nutrición es un escenario propicio para alcanzar este objetivo.

\section{AGRADECIMIENTOS}

A los pacientes por su participación, a las instituciones por permitir el estudio, a la nutricionista Amida Carvajal Guzmán por su apoyo y a la Institución Universitaria Escuela Nacional del Deporte por su compromiso con la investigación.

\section{DECLARACIÓN SOBRE CONFLICTO DE INTERESES}

Como autores declaramos no tener conflicto de intereses. 


\section{CONTRIBUCIÓN DE LOS AUTORES}

De acuerdo a las normas nacionales e internacionales que regulan los derechos de autor en una publicación científica, todos los autores declaran tener participación activa en dos o más de los siguientes ítems:

a) Participación sustantiva en la concepción, diseño, análisis e interpretación de los datos.

b) Redacción del borrador del artículo, análisis y revisión crítica de sus contenidos intelectuales.

c) Aprobación de la versión final a ser publicada.

\section{REFERENCIAS BIBLIOGRÁFICAS}

1. World Health Organization. Noncommunicable diseases [internet] [citado 05 abr 2018]. Disponible en: https://www.who.int/newsroom/fact-sheets/detail/noncommunicablediseases.

2. International Diabetes Federation. Diabetes Atlas. Eighth edition 2017 [internet] [citado 05 abr 2018]. Disponible en: http://fmdiabetes.org/atlas-idf-2017/.

3. World Health Organization. World Health Statistics 2018: Monitoring health for the SDGs [internet] [citado 17 sep 2018]. Disponible en: https://www.who.int/gho/publications/world _health_statistics/2018/en/.

4. World Health Organization. Global recommendations on physical activity for Health [internet] [citado 05 abr 2018]. Disponible en: https://apps.who.int/iris/bitstream/handle/10 665/44399/9789241599979_eng.pdf?sequenc $\mathrm{e}=1$.

5. American Diabetes Association. Standards of Medical Care in Diabetes 2018 [internet] [citado 07 abr 2018]. Disponible en: http://care.diabetesjournals.org/content/diac are/suppl/2017/12/08/41.Supplement_1.DC1/ DC_41_S1_Combined.pdf.

6. Sapunar J. Epidemiología de la Diabetes Mellitus en Chile. Rev Med Clín Las Condes [revista en la internet]. 2016 [citado 26 nov 2018]; 27(2): 146-51. Doi: 10.1016/j.rmclc.2016.04.003.

7. Leiva T, Basfi-fer K, Rojas P, Carrasco F, Ruz M. Efecto del fraccionamiento de la dieta y cantidad de hidratos de carbono en el control metabólico en pacientes con diabetes mellitus tipo 2, sin terapia con insulina. Rev Méd de Chile [revista en la internet]. 2016 [citado 26 nov 2018]; 144(10): 1247-53. Doi: http://dx.doi.org/10.4067/S003498872016001000002.

8. Instituto Nacional de Estadística e Informática. Encuesta Demográfica y de Salud Familiar ENDES 2014 [internet] [citado 16 oct 2018]. Disponible en: https://www.inei.gob.pe/media/MenuRecursi vo/publicaciones_digitales/Est/Lib1211/pdf/Li bro.pdf.

9. Instituto Colombiano de Bienestar Familiar. Encuesta nacional de situación nutricional en Colombia ENSIN 2010 [internet] [citado 17 sept 2018]. Disponible en: https://www.icbf.gov.co/bienestar/nutricion/e ncuesta-nacional-situacion-nutricional.

10. Ministerio de Salud de Chile, Instituto de Salud Pública de Chile. Encuesta Nacional de Consumo de Alimentos 2010-2011 [internet] [citado 17 sept 2018]. Disponible en: http://www.ispch.cl/noticia/15048.

11. Secretaría de Salud de México. Encuesta Nacional de Salud y Nutrición de Medio Camino 2016 [internet] [citado 17 sept 2018]. Disponible en: https://www.gob.mx/salud/documentos/encu esta-nacional-de-salud-y-nutricion-de-mediocamino-2016.

12. Bowman, BA. Russell, MR. Conocimientos actuales sobre nutrición [internet]. Octava edición. Washington: Organización Panamericana de la Salud № 592; 2003 [citado 17 sept 2018]. Disponible en: https://es.scribd.com/document/69390923/C onoc-Actuales-Sobre-Nutricion.

13. Vague J. Degree of masculine differentiation of obesity: factor determining predisposition to 
diabetes, atherosclerosis, gout, and uric calculus disease. Am J Clin Nutr Condes [revista en la internet]. 1956 [citado 26 nov 2018]; 4: 20-34. Doi: 10.1093/ajcn/4.1.20.

14. King, H. Gruber, W. Lander, T, World Health Organization. Division of Noncommunicable Diseases. Implementing national diabetes programs: report of a WHO meeting [internet]. 1995 [citado 17 sept 2018]. Disponible en: http://www.who.int/iris/handle/10665/60618.

15. American Diabetes Association. Diagnosis and Classification of Diabetes Mellitus. Diabetes Care [revista en la internet]. 2005 [citado 25 agos 2018]; 28: S37-S42. Disponible en: https://doi.org/10.2337/dc10-S062.

16. Gadsby R. Epidemiology of diabetes. Adv. Drug Deliv. Rev [revista en la internet]. 2002 [citado 26 nov 2018]; 54: 1165-72. Disponible en: https://doi.org/10.1016/S0169409X(02)00094-7.

17. King $H$, Aubert R, Herman W. Global Burden of diabetes,1995-2025. Prevalence, numerical estimates, and projections. Diabetes Care [revista en la internet]. 1998 [citado 26 agos 2018]; 21: 1414-1431. Disponible en: https://doi.org/10.2337/diacare.21.9.1414.

18. Villena J. Diabetes Mellitus in Peru. Annals of Global Health [revista en la internet]. 2015 [citado 26 nov 2018]; 81: 765-775. Disponible en:

https://doi.org/10.1016/j.aogh.2015.12.018.

19. Díaz-Martínez, X. Petermann, F. Leiva, AM. Garrido-Méndez, A. Salas-Bravo, C. Martínez, $\mathrm{MA}$, et al. Association of physical inactivity with obesity, diabetes, hypertension and metabolic syndrome in the Chilean population. Rev Med Chil [revista en la internet]. 2018 [citado 29 nov 2018]; 146(5): 585-595. Doi: 10.4067/s003498872018000500585 .

20. Organización Mundial de la Salud. Informe sobre la salud en el mundo 2002 [internet] [citado 26 agos 2018]. Disponible en: https://www.who.int/whr/2002/es/.

21. Organización Mundial de la Salud. Detener la Epidemia mundial de las enfermedades crónicas [internet] [citado 08 feb 2018].
Disponible en: https://www.paho.org/hq/dmdocuments/201 2/OPS-Detener-epidemia-mundial-EC2006.pdf.

22. Organización Mundial de la Salud. Informe sobre la situación mundial de las enfermedades no transmisibles 2010 Resumen de Orientación [internet] [citado 17 sept 2018]. Disponible en: https://www.who.int/nmh/publications/ncd_r eport2010/es/.

23. Ministerio de Salud y Protección Social. Encuesta Nacional de Salud. ENS 2010 [internet] [citado $07 \mathrm{abr}$ 2018]. Disponible en: http://www.minproteccionsocial.gov.co/VBeC ontent/library/documents/DocNewsNo18358 DocumentNo9089.PDF.

24. Arsenault BJ, Boekholdt SM, Kastelein JJ. Lipid parameters for measuring risk of cardiovascular disease. Nat Rev Cardiol [revista en la internet]. 2011 [citado 29 nov 2018]; 8: 197-206. Doi: 10.1038/nrcardio.2010.223.

25. Zalesin KC, Franklin BA, Miller WM, Peterson ED, McCullough PA. Impact of obesity on cardiovascular disease. Med Clin North Am [revista en la internet]. 2011 [citado 29 nov 2018]; 95: 919-937. Doi: 10.1016/j.mcna.2011.06.005.

26. Admiraal WM, van Valkengoed IG, L de Munter JS, Stronks K, Hoekstra JB, Holleman F. The association of physical inactivity with Type 2 diabetes among different ethnic groups. Diabet Med [revista en la internet]. 2011 [citado 30 nov 2018]; 28: 668-672. Doi: 10.1111/j.14645491.2011.03248.x.

27. Bell J, George W, García M, Delgado E, Bell M. Identificación del síndrome metabólico en pacientes con diabetes mellitus e hipertensión arterial. Medisan [revista en la internet]. 2017 [citado 29 nov 2018]; 21(10): 3038-45. Disponible en: http://scielo.sld.cu/scielo.php?script=sci_artte xt\&pid=S1029-30192017001000007.

28. Organización Mundial de la Salud. Información general sobre hipertensión en el mundo: una enfermedad que mata en silencio, una crisis de salud pública mundial [internet] [citado 02 agos 
2019].

Disponible

en:

http://apps.who.int/iris/bitstream/10665/876

79/1/WHO_DCO_WHD_2013.2_spa.pdf.

29. Consenso Latinoamericano de la Asociación Latinoamericana de Diabetes. Epidemiología, diagnóstico, control, prevención y tratamiento del síndrome metabólico en adultos. Rev Asoc Latinoam Diab [revista en la internet]. 2010 [citado 02 agos 2019]; 18(1): 25-44. Disponible en: http://www.revistaalad.com/pdfs/10012544.pdf.

30. Won KB, Chang HJ, Kim HC, Jeon K, Lee H, Shin $\mathrm{S}$, et al. Differential impact of metabolic syndrome on subclinical atherosclerosis according to the presence of diabetes. Cardiovasc Diabetol [revista en la internet]. 2013 [citado 01 agos 2019]; 12:41. Doi: 10.1186/1475-2840-12-41.
31. D'Agostino RB, Vasan RS, Pencina MJ, Wolf PA, Cobain M, Massaro JM, et al. General Cardiovascular Risk Profile for Use in Primary Care. The Framingham Heart Study. Circulation. Epub [revista en la internet]. 2008 [citado 29 nov 2018]; 117: 743-753. Doi: 10.1161/CIRCULATIONAHA.107.699579.

32. Grundy SM, Becker D, Clark LT, Cooper RS, Denke MA, Howard WJ., et al. Third Report of the National Cholesterol Education Program (NCEP) Expert Panel on Detection, Evaluation, and Treatment of High Blood Cholesterol in Adults (Adult Treatment Panel III) NIH Publication No. 02-5215. Circulation [revista en la internet]. 2002 [citado 29 nov 2018]; 106: 3143. Disponible en: https://doi.org/10.1161/circ.106.25.3143. 